\title{
Protocolos de Aplicação de Toxina para Sorriso Gengival: uma revisão de literatura
}

\author{
Protocols for the Application of Toxin for Gengival Smiling: a review of literature \\ Protocolos de Aplicación de Toxina para Sonrisa Gengival: una revisa de literatura
}

José Milton de Aquino e Silva Neto ${ }^{1 *}$, Andressa Rebecca Coêlho Batista', Íris Régia Ventura Barros ${ }^{1}$, Yasmin Bitencourt Montenegro de Araujo', Isabel Karine Ferreira Duarte ${ }^{1}$, João Francisco Tenório Neto ${ }^{1}$.

\section{RESUMO}

Objetivos: Este estudo foi obtido através de uma revisão de literatura envolvendo os protocolos de aplicação de toxina botulínica para o sorriso gengival, investigando a indicação deste material para a harmonia do sorriso, analisando os protocolos desta aplicação e determinando o porquê da utilização das toxinas botulínicas ao invés da cirurgia. Métodos: Trata-se de uma revisão bibliográfica, realizada através de uma abordagem qualitativa. Entre os principais parâmetros para o levantamento bibliográfico, destaca-se o acervo de obras online obtidos na Scientific Eletronic Library Online (SCIELO), nas bases de dados Literatura Latino Americano em Ciências da Saúde (LILACS), Biblioteca Virtual de Saúde (BVS) e no Google Acadêmico. Resultados: Com o propósito de acabar com o problema do sorriso gengival e devolver ao paciente um sorriso harmônico e aprazível, destacase o uso da toxina botulínica, por ser uma alternativa mais viável, segura, eficiente e menos invasiva, quando comparada à cirurgia reparadora. Conclusão: A toxina botulínica é uma ótima opção para solucionar o problema de sorriso gengival, por ser de fácil aplicação e menos invasiva.

Palavras-chave: Protocolo, Toxina Botulínica, Sorriso Gengival.

\begin{abstract}
Objectives: This study was obtained through a literature review involving the botulinum toxin application protocols for the gingival smile, investigating the indication of this material for the smile harmony, analyzing the protocols of this application and determining the use of botulinum toxins instead surgery. Methods: This is a bibliographical review, carried out through a qualitative approach. Among the main parameters for the bibliographic survey, the collection of online works obtained in the Scientific Electronic Library Online (SCIELO), the Latin American Literature in Health Sciences (LILACS), the Virtual Health Library (VHL) and on Google Scholar. Results: In order to eliminate the problem of gingival smile and give the patient a harmonious and pleasant smile, the use of botulinum toxin is highlighted, since it is a more viable, safe, efficient and less invasive alternative when compared to surgery restorative Conclusion: Botulinum toxin is a great option to solve the problem of gingival smile, because it is easy to apply and less invasive.
\end{abstract}

Key words: Protocol, Botulinum Toxin, Gingival Smile.

\section{RESUMEN}

Objetivos: Este estúdio se ha obtenido a través de una revisión de literatura envolviendo los protocolos de aplicación de toxina botulínica para la sonrisa gingival, investigando la indicación de este material para la armonía de la sonrisa, analizando los protocolos de esta aplicación y determinando el por qué de la utilización de las toxinas botulínicas en lugar de la cirugía. Métodos: Se trata de una revisión bibliográfica, realizada a través de un abordaje cualitativo. Entre los principales parámetros para el levantamiento bibliográfico, se destaca el acervo de obras online obtenidas en la Scientific Eletronic Library Online (SCIELO), en las bases de datos Literatura Latinoamericana en Ciencias de la Salud (LILACS), Biblioteca Virtual de Salud (BVS) en Google

${ }^{1}$ Centro Universitário CESMAC (CESMAC), Maceió-Alagoas. *E-mail: Milton neto 166@hotmail.com

\begin{tabular}{l|l|l|} 
SUBMETIDO EM: 6/2019 | ACEITO EM: 7/2019 | PUBLICADO EM: 7/2019
\end{tabular} 
Académico. Resultados: Con el propósito de acabar con el problema de la sonrisa gingival y devolver al paciente una sonrisa armónica y apacible, se destaca el uso de la toxina botulínica, por ser una alternativa más viable, segura, eficiente y menos invasiva, cuando comparada a la cirugía restauradora. Conclusión: La toxina botulínica es una gran opción para solucionar el problema de la sonrisa gingival, por ser de fácil aplicación y menos invasiva.

Palabras clave: Protocolo, Toxina Botulínica, Sonrisa Gingival.

\section{INTRODUÇÃO}

A busca por um sorriso perfeito tem sido a nova tendência em relação aos padrões de beleza. Sendo assim, a harmonia da estética facial encontra-se relacionada diretamente com o sorriso. O sorriso é formado pela união dos três elementos: dentes, gengivas e lábios. Por tanto, para que haja um aspecto agradável no ato de sorrir de um indivíduo, é ressaltado a importância de haver uma disposição adequada destes elementos, neste sentido a toxina botulínica surge como sendo um eficaz auxiliar para o tratamento de diversos problemas de ordem odontológica, podendo ser utilizada tanto na área da cosmetologia, quanto para fins terapêuticos. Dentre eles, destacam-se o bruxismo, disfunção temporomandibular, hipertrofia do masseter e exposição gengival acentuada (PEDRON IG, 2014).

Percebe-se que a quantidade de gengiva aparente é motivo de descontentamento para muitas pessoas, uma vez que é uma condição facilmente detectada pelo próprio paciente. Este problema é denominado de sorriso gengival e se caracteriza por uma excessiva exposição da gengiva sendo uma das queixas frequentes dos pacientes no decorrer da anamnese odontológica (OLIVEIRA MT e MOLINA RO, 2011). Inicialmente, a toxina botulínica era utilizada somente na medicina, com fins terapêuticos. No entanto, após algumas pesquisas, passou a ser aplicada também nos tratamentos estéticos na odontologia, mostrando-se ser muito eficiente (MATOS MB, et al., 2017).

Convém ressaltar que sua utilização nesta área ainda é muito recente. Esta substância é produzida pela bactéria anaeróbia denominada Clostridium botulinum, causadora do botulismo. Ela passa por um processo industrializado que a purifica e a deixa apta a ser usada em doses adequadas que não fazem mal à saúde (OLIVEIRA MT e MOLINA RO, 2011).

Quando é realizado o diagnóstico do sorriso gengival em um paciente, a toxina botulínica apresenta-se como uma especificidade no tratamento, por ser uma técnica minimamente invasiva, onde na maioria dos casos serve como forma de auxiliar ou substituir o procedimento cirúrgico. Esta toxina age aderindo-se à proteína sinaptosômica (SNAP-25) que por sua vez inibi a liberação de acetilcolina, impossibilitando, desse modo, a contração muscular. Existem sete tipos de neurotoxina botulínicas sorologicamente acessível, porém a toxina botulínica tipo A é com mais frequência aplicada (KEANEY TC e ALSTER TS, 2013).

Este artigo tem como objetivo analisar os protocolos utilizados na toxina botulínica que vem sendo empregados na odontologia, baseando-se principalmente no aspecto do melhoramento do sorriso gengival, a fim de analisar a indicação deste material para a harmonia do sorriso, levando em consideração os protocolos de aplicação para identificar o porquê da utilização das toxinas botulínicas ao invés da cirurgia, tendo em vista que a toxina botulínica tem se mostrado bastante eficiente como recursos terapêuticos no que se refere ao sorriso gengival, principalmente em pacientes com hiperfunção dos músculos envolvidos no sorriso, bem como em outras desordens como é o caso das disfunções temporomandibulares e a dor miofascial.

\section{MÉTODOS}

Esta pesquisa constituiu-se através de uma revisão de literatura realizada entre os períodos de fevereiro de 2019 a junho de 2019, onde foram analisados artigos publicados em bases de dados eletrônicos (Pubmed, Mesh, portal de periódicos CAPES, EBSCO, DeCS, e Google Acadêmico) e livros que estavam disponíveis nos acervos bibliográficos presentes na biblioteca central do Centro Universitário Cesmac. 
Foram consultados textos em língua portuguesa e inglesa, que se referiram aos protocolos de aplicação no uso da toxina botulínica, tendo como critérios de exclusão os períodos de publicações e os artigos que não possuíam relevância com a temática, sendo selecionados os textos científicos que apresentavam na integra o real objetivo do trabalho, verificando a presença de mudanças e avanços no estudo da toxina botulínica para uma melhor harmonização do sorriso gengival. Os descritores utilizados foram: protocolos de aplicação da toxina botulínica, crescimento excessivo da gengiva e estética dentária.

\section{RESULTADOS E DISCUSSÕES}

Durante o século XVII, na Europa, ocorreram muitas mortes causadas pela ingestão do Clostridium botulinum, os quais se encontravam presentes em alimentos contaminados. Devido essas intercorrências no ano de 1822, o alemão Justinius Kerner, deu início ao estudo sobre a toxina botulínica, sendo o primeiro pesquisador a fazer referência ao envenenamento por salsicha, devido ao fato dela ser a causa da intoxicação. Desta maneira, no ano de 1871, o termo botulus foi utilizado para nomear esta doença. Com o aumento dos casos desta intoxicação ao longo do tempo, a Dole Food Company Inc, observando o grande número de ocorridos, desenvolveu novas tecnologias para enlatar os alimentos, que possibilitassem uma conservação e manuseamento seguro para a saúde" (MATOS MB, et al., 2017).

Neste sentido, em 1981, estudos foram realizados por Alan Scott, no qual ele descreveu uma experiência com o uso da toxina botulínica tipo A (BTX-A), onde foi demostrado as suas características, composição, fabricação, sendo os resultados obtidos em macacos e em seres humanos com sua aplicação em músculos extraoculares, apresentando, por sua vez, resultados benéficos como uma modalidade terapêutica. Assim, a BTX ficou sendo conhecida por ser usada no âmbito da medicina estética, com o intuito de diminuir os sinais de envelhecimento. Entretanto, ela é extremamente importante devido a suas propriedades terapêuticas, sendo aprovada pela ANVISA no Brasil em 2000 (MATOS MB, et al., 2017).

A BTX é um agente biológico produzido, de forma natural, pela bactéria anaeróbica gram positiva Clostridium botulinum, que ao ser aplicada na musculatura, dirigi-se para as terminações nervosas, ocasionando o bloqueio dos canais de cálcio, coibindo a liberação de acetilcolina e por consequência desse mecanismo promovendo o relaxamento ou paralisia do músculo alvo de forma transitória (MOCK D, 2015).

O seu restabelecimento fisiológico normalmente acorre gradativamente, após dois a três meses de sua aplicação. Assim, observa-se que a reversão da paralisia acontece por meio de dois mecanismos que são, o brotamento neural, onde ocorre o desenvolvimento dos brotos axonais e a reinervação e formação de novas placas terminais menores. A reinervação muscular acontece pela regeneração de proteínas de acoplamento das vesículas de acetilcolina cuja função geralmente é restabelecida entre um e quatro meses (CALZA SC, et al., 2015).

De acordo com a literatura, a BTX tem sido estudada há mais de trinta anos. Seu início deu-se em Fort Detrick, e após, na Universidade de Wisconsin, onde foi produzida laboratorialmente pela primeira vez. Na área odontológica, seu uso foi devidamente regulamentado através da Resolução 112/11 do Conselho Federal de Odontologia. Na odontologia, a toxina começou a ser empregada na correção do sorriso gengival sendo utilizada desta forma para evitar meios mais invasivos (BRASIL, 2011).

Levando-se em consideração que um sorriso esteticamente acurado deve obedecer algumas condições, tais como, a posição labial superior, deve-se considerar a altura de um sorriso em particular, diferenciando-se de um paciente para outro. Sendo assim, um diagnóstico adequado deve ser realizado por um profissional capacitado que leve em consideração o sexo, idade e saúde periodontal, e que também observe o tipo de anormalidade, determinando desta forma, os fatores etiológicos em cada situação e qual a estratégia de tratamento mais apropriada deve ser tomada (MACELO ACV, et al., 2012).

Do mesmo modo, é de vital importância que ocorra a análise dentária em toda sua conceituação quanto ao plano oclusal do paciente, bem como o exame do lábio superior, devendo o mesmo ser mantido ao nível da margem gengival dos incisivos centrais superiores, levando-se em consideração que uma exposição gengival 
em um sorriso que atinge $4 \mathrm{~mm}$ é considerado como sendo um sorriso antiestético, e por tanto a sua relevância frente ao diagnóstico e tratamento para que se contribua com a correção do sorriso gengival. (SENISE IR, et al., 2015).

Dentro da dinâmica do sorrir, os principais músculos envolvidos no sorriso são o elevador do lábio superior, asa do nariz, o músculo zigomático maior e zigomático menor. Cada músculo envolto na elevação do lábio superior, possui uma determinada função quando se sorri. Desta forma, observa-se que cada tipo de sorriso gengival acaba envolvendo um determinado músculo (SPONITO MMM, 2004).

As figuras 1 e 2 apresentam os músculos envolvidos na expressão facial do sorriso no indivíduo. É pertinente considerar que cada musculatura responsável pelo levantamento do lábio superior expressa uma função particular durante a atividade do sorriso, e para que se possa aplicar a toxina precisa-se saber cada classe de sorriso gengival envolvido, determinado assim, quais os músculos envolvidos e o método de aplicação (MADEIRA MC, 2001).

\begin{tabular}{|l|l|}
\hline $\begin{array}{l}\text { Figura 1 - Músculos responsáveis pelo sorriso } \\
\text { espontâneo }\end{array}$ & Figura 2 - Músculos responsáveis pela gargalhada \\
\hline 6 & 5
\end{tabular}

Fonte: Desenho realizado por (SILVA NETO JMA, et al., 2019), baseado nos dados do livro (MADEIRA MC, 2001) e (FLÁVIO A, 2019).

Há vários tipos de tratamento de correção do sorriso gengival. Contudo, alguns demandam certo receio por parte dos pacientes que, muitas vezes, acabam desistindo do tratamento, principalmente quando envolve um procedimento cirúrgico. Dentre os protocolos utilizados nestes casos, tem-se a cirurgia de reposicionamento dos músculos labiais e o uso de toxina botulínica, que relaxa a musculatura dos lábios superiores (MATOS MB, et al., 2017).

Assim, Rego RV, et al (2015); e Magro AKD, et al (2015); afirmam que, com o propósito de dirimir o problema da exposição de excesso de gengiva e devolver ao paciente um sorriso harmônico e aprazível, surgem algumas opções odontológicas que podem corrigir ou minimizar o sorriso gengival. Para os autores, a toxina botulínica é uma alternativa mais viável, segura, eficiente e menos invasiva, quando comparada à cirurgia reparadora.

A utilização da toxina botulínica é um recurso rápido e seguro, podendo ocasionar um resultado harmonioso e agradável quando aplicado em músculos-alvos, ou seja, no elevador do lábio superior e da asa do nariz e zigomáticos maior e menor, como pode ser visto na figura 3. Contudo o autor enfatiza a importância de se respeitar a dose apropriada e o tipo de sorriso do paciente (PEDRON IG, 2014).

Figura 3 - Demarcação dos locais de punção. 


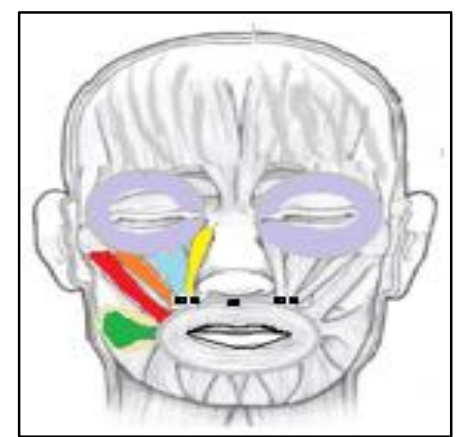

Fonte: Desenho realizado por (SILVA NETO JMA, et al., 2019), baseado nos dados do livro (MADEIRA MC, 2001) e (FLÁVIO A, 2019).

Quanto as marcações dos pontos de punção, estas são realizadas com o lápis dermográfico apontado. Os músculos devem estar em repouso para as aplicações. A dosagem recomendada é de $2 \mathrm{U}$ a $3 \mathrm{U}$ por ponto para pessoas com 3 a $5 \mathrm{~mm}$ de exposição gengival. O conteúdo deve ser dividido igualmente para cada um dos lados e aplicado por meio de uma agulha $4 \mathrm{~mm}$ em uma direção oblíqua à superfície da pele. Conforme a literatura, as injeções devem ser realizadas delicadamente, com uma leve pressão sobre o êmbolo. Recomenda-se o uso de gelo após o procedimento a fim de se evitar edemas e hematomas. Nesse contexto, a BTX-A é injetada dentro dos músculos encarregados na elevação do sorriso por meio de finas agulhas, provocando a fraqueza muscular, e assim atenuando a expressão e a assimetria. Sua aplicação em um determinado local, faz com que o músculo não se contraia, mantendo-se relaxado (BARBOSA CMR e BARBOSA JRA, 2017).

A toxina botulínica possui propriedades altamente imunogênica, com isso ocorre o desenvolvimento e formação de anticorpos. Se o profissional aplicar doses elevadas na administração ou ocorrer aplicações com frequência sem respeitar os intervalos de tempo, ocorrerá a diminuição da função da toxina com o passar do tempo. Em diversas ocasiões a mudança para outro subtipo de toxina, pode ser imprescindível. A toxina tipo $B$ é menos utilizada nos procedimentos quando comparada ao tipo $A$, devido este tipo ser mais imunogênica, ou seja, na obtenção de um resultado satisfatório exige-se doses cada vez maiores, acarretando uma maior carga de proteínas e por efeito elevando sempre a mais seu potencial imunogênico (DUTRA MB, et al., 2011).

Para que o uso estético da TXB-A seja assegurado, faz-se necessário a administração de pequenas aplicações que duram aproximadamente seis meses, sendo prescrita sua reaplicação para que os efeitos permaneçam. A toxina botulínica vem sendo a opção preferida tanto pelos profissionais da área, como para os pacientes, uma vez que acaba por favorecer ambas as partes. Sua utilização é mais indicada por ser um método menos invasivo que a cirurgia e, consequentemente minimiza o tempo de recuperação. No entanto, ela também pode ser usada em casos de implantodontia, sorriso torto, bruxismo entre outros. (PEDRON IG, 2014).

Para sua administração é necessário providenciar um frasco de $10 \mathrm{~mL}$ de soro fisiológico estéril a $0,9 \%$, uma seringa estéril de $2 \mathrm{~mL}$ e uma agulha estéril de $25 \mathrm{~mm} / 22 \mathrm{G}$. Na aplicação, são usados uma seringa estéril de $1 \mathrm{~mL}$ com resíduo zero, 1 agulha de $6 \mathrm{~mm} / 32 \mathrm{G}$, gaze estéril, luvas de procedimento estéreis, algodão estéril, gelo, solução de clorexidine a $2 \%$, lápis dermográfico branco para a marcação das áreas de segurança e/ou dos pontos de aplicação, campo cirúrgico estéril e anestésico tópico (EMLA® - lidocaína a 2,5\%: prilocaína a 2,5\%). O paciente deve encontrar-se sentado de forma confortável na cadeira do profissional a uma inclinação de 60 . Em seguida, é realizada a antissepsia dos locais de aplicação com a clorexidine $2 \%$, após a instalação do campo cirúrgico estéril, sem encobrir a face. Em torno de 15 a 20 minutos antes de dar início à aplicação da toxina botulínica, $2 \mathrm{~g}$ do anestésico tópico são aplicadas com algodão estéril sobre as áreas da punção. $\mathrm{A}$ demarcação dos locais de punção será realizada solicitando-se ao paciente que contraia o lábio superior, como em um sorriso forçado. (BARBOSA CMR e BARBOSA JRA, 2017).

Tabela 1 - Demonstração esquematizada da dosagem na aplicação para a obtenção do resultado esperado tanto do profissional quanto do paciente (MADEIRA MC, 2001). 


\begin{tabular}{|l|c|c|c|c|}
\hline MÚSCULO & $\begin{array}{l}\text { PROFUNDIDADE } \\
\text { DA AGULHA }\end{array}$ & $\begin{array}{l}\text { PONTOS } \\
\text { POR } \\
\text { MÚSCULO }\end{array}$ & $\begin{array}{l}\text { UNIDADES } \\
\text { POR PONTO }\end{array}$ & $\begin{array}{l}\text { TOTAL DE } \\
\text { UNIDADE/MÚSCULO }\end{array}$ \\
\hline $\begin{array}{l}\text { Levantador do } \\
\text { ângulo da boca } \\
\text { (Direito) }\end{array}$ & $4 \mathrm{~mm}$ & 1 & $3 \mathrm{U}$ & $3 \mathrm{U}$ \\
\hline $\begin{array}{l}\text { Levantador do } \\
\text { ângulo da boca } \\
\text { (Esquerdo) }\end{array}$ & $4 \mathrm{~mm}$ & 1 & $3 \mathrm{U}$ & $3 \mathrm{U}$ \\
\hline $\begin{array}{l}\text { Levantador do } \\
\text { lábio superior e } \\
\text { da asa do nariz } \\
\text { (Direito) }\end{array}$ & $4 \mathrm{~mm}$ & 1 & $3 \mathrm{U}$ & \\
\hline $\begin{array}{l}\text { Levantador do } \\
\text { lábio superior e } \\
\text { da asa do nariz } \\
\text { (Esquerdo) }\end{array}$ & $4 \mathrm{~mm}$ do & 1 & $3 \mathrm{U}$ \\
\hline $\begin{array}{l}\text { Depressor } \\
\text { septo nasal do }\end{array}$ & $8 \mathrm{~mm}$ & 1 & $3 \mathrm{U}$ \\
\hline
\end{tabular}

Fonte: Próprio autores, 2019. Modificada de (MADEIRA MC, 2001).

É importante atentar-se quanto as precauções necessárias a serem tomadas no que diz respeito a aplicação da toxina botulínica. Mesmo tendo o conhecimento de ser um procedimento simples e seguro, pode ocorrer alguns sintomas adversos, tais como: dor no local onde foi efetuada a aplicação, possíveis hematomas, se o paciente e o profissional não tomar os devidos cuidados pode ocorrer infecção, edemas, breve disfonia acompanhada de disfagia, queda da pálpebra superior podendo ser uni ou bilateral, alongamento do lábio superior e assimetria do sorriso.

É também de suma importância ressaltar que nas primeiras 24 horas após a aplicação, $60 \%$ da substância encontrada na toxina é excretada pela urina. O Cirurgião Dentista especializado deve informar ao paciente os cuidados, pós aplicação da BTX- A, que devem ser tomados: evitar massagear a área onde foi realizado o procedimento logo após a aplicação; instruir para que o mesmo se mantenha em posição vertical, ou seja, não se deitando durante as primeiras horas logo após a aplicação da toxina e não fazer exercícios físicos no decorrer das primeiras 24 horas após aplicação (PANOSSIAN AJ e BLOCK MS., 2010).

Apesar do uso desta toxina não oferecer riscos de um modo geral, convém ressaltar que mulheres grávidas ou amamentando e pessoas alérgicas ao produto não devem fazer uso deste tipo de tratamento. Outros casos em que a toxina botulínica não deve ser usada são: neuropatia muscular, desordem muscular como esclerose lateral amiotrófica (ELA), síndrome de Lambert Eaton, Distrofia Muscular, Esclerose Múltipla e quem faz uso de bloqueadores de canais de cálcio e amino glicosídeos (SENISE IR, et al., 2015).

\section{CONSIDERAÇÕES FINAIS}

Dentre os protocolos utilizados em casos de sorriso gengival, o tratamento com a toxina botulínica tornouse uma ótima opção para pacientes que apresentam este problema, por ser menos invasivo que o procedimento cirúrgico. Este produto é de fácil aplicação, apresentando poucas contraindicações e complicações, porém com curto espaço de duração, tonando-se oneroso.

Com base na literatura, percebe-se que a maioria dos autores concorda que o fator decisório para o uso deste produto é a etiologia apresentada pelo paciente. Contudo, observa-se uma escassez de estudos envolvendo o assunto. Assim, sugere-se que mais pesquisas randomizadas sejam efetuadas, a fim de assegurar a eficácia e a convicção da aplicação da toxina botulínica envolvida nos tratamentos de casos de sorriso gengival.

\section{REFERÊNCIAS}


1. BARBOSA CMR, BARBOSA, JRA. Toxina botulínica em odontologia. 1ed. Rio de Janeiro: Elsevier Edit Ltda Conhecimento sem Fronteiras, 2017; 64p.

2. BRASIL. Conselho Federal de Odontologia (CFO). Resolução 112/11 n 171, de 2 de setembro de 2011. Baixa normas sobre a utilização do uso da toxina botulínica e ácido hialurônico. 2011. Disponível em: http://cromg.org.br/arquivos/Resolucao\%20CF0112-2011.pdf. Acesso em: 21 mai. 2019.

3. CALZA SC. et al. Tratamento do sorriso gengival com toxina botulínica tipo A: relato de caso. RFO UPF, 2015; 20(10): 81-87.

4. DUTRA MB, et al. Influência da exposição gengival na estética do sorriso. Dental Press J Orthod 2011; 16: 5111 -18.

5. FLÁVIO A. Toxina Botulínica para harmonização facial. 1 ed. São Paulo: Napoleão Ltda; 2019; 78-95.

6. KEANEY TC, ALSTER TS. Botulinum toxin in men: review of relevant anatomy and clinical trial data. World Environment Day 2013; 39(10):1434-43.

7. MACEDO ACVB, et al. O sorriso gengival-tratamento baseado na etiologia: uma revisão de literatura. Revista Periodontia, 2012; 22(4): 36-44.

8. MADEIRA MC. Anatomia da face: bades anátomo-funcionais para a prática odontológica. 3 ed. São Paulo: SARVIER, $2001 ; 67-96$

9. MAGRO AKD. Tratamento do sorriso gengival com toxina botulínica tipo A: relato de caso. RFO, Passo Fundo, 2015; 20(1): 81-87, jan./abr.

10. MATOS MB, et al. O uso da toxina botulínica na correção do sorriso gengival - revisão de literatura. Braz J Periodontol, 2017; 27: 29-36.

11. MOCK D, et al. Botulinum Toxin and Dentistry. In: Ensuring Continued Trust. Royal College of Dental Surgeons of Ontario, Toronto, 2009; 1-4.

12. OLIVEIRA MT, MOLINA RO. Sorriso Gengival, quando a toxina botulínica pode ser utilizada. Rev. Odontol. Araçatuba, 2011; 32 (2): 58-61.

13. PANOSSIAN AJ, BLOCK MS. Evaluation of the Smile: Facial and Dental Considerations. J Oral Maxillofac Surg 2010; 68: 547-554

14. PEDRON IG. Cuidados no planejamento para a aplicação da Toxina Botulínica em Sorriso Gengival. Revista Odontol. São Paulo: UNICID; 2014; 26 (3): 250-6.

15. REGO RV, et al. Complementation of resective peiodontal sugery by aplication of botulinum toxin in the management of gummy smile in orthodontic patient. Orthod. Sci. Pract. 2015; 8 (29): 74-78.

16. SENISE IR, et al. O uso de toxina botulínica como alternativa para o tratamento do sorriso gengival causado pela hiperatividade do lábio superior. Maringá: Revista UNINGÁ Review, 2015; 23(3): 104-110.

17. SPOSITO MMM. Toxina botulínica tipo A - propriedades farmacológicas e uso clínico. Acta Fisiátrica, $2004 ; 1:$ 7-44. 